Check for updates

Cite this: Chem. Sci., 2019, 10, 227

๑ All publication charges for this article have been paid for by the Royal Society of Chemistry

Received 16th July 2018

Accepted 29th September 2018

DOI: $10.1039 / \mathrm{c} 8 \mathrm{sc} 03135 \mathrm{e}$

rsc.li/chemical-science

\section{Controllably realizing elastic/plastic bending based on a room-temperature phosphorescent waveguiding organic crystal $\dagger$}

\author{
Hao Liu, Zhengyi Bian, Qinyu Cheng, Linfeng Lan, Yue Wang (D) \\ and Hongyu Zhang (iD *
}

Recently, the study of flexible (elastically bendable and plastically bendable) organic single crystals has become a hot research field in crystal engineering. In general, crystal elasticity and plasticity are incompatible with each other. Different from the applications of fluorescent crystals, the applications of room-temperature phosphorescence (RTP) materials generally ignore the crystallographic nature of large single crystals. Herein, we creatively combine elasticity and plasticity based on one RTP crystal $4,4^{\prime}$-dibromobenzil DBBZL. The in-depth study of the irreversible transformation between elastic bending and plastic bending provided important insights into the mechanism of both elastically bendable crystals and plastically bendable crystals in crystal engineering. The DBBZL crystal exhibits elastic bending (reversible) under external stress, whereas it shows plastic bending (irreversible) after excessive bending. Notably, the first phosphorescent optical waveguides of large single RTP crystals are realized not only in straight state, but also in elastic bent state and plastic bent state.

\section{Introduction}

Nowadays, the intersection of crystal engineering and functional material has become a developing trend of advanced materials. ${ }^{1-18}$ The mechanical behaviors of organic crystals such as bending ${ }^{6-14}$ and twisting ${ }^{15-18}$ have become hot topics in crystal engineering. As these mechanical properties endow organic crystals with flexible structures, they may exhibit the same functions in a complex environment. ${ }^{19-22}$ Among these materials, flexible crystals with reversible bending (elasticity) or irreversible bending (plasticity) are of core interest because of potential applications in many fields..$^{23-25}$ However, the flexible crystal species are still very rare. The reason is that the structures of flexible crystals should be well designed. Ghosh et al. proposed that the packing structures of one-dimensional (1D) elastically bendable crystals should be interlocked with weak and dispersive interactions, ${ }^{26}$ while the $1 \mathrm{D}$ plastically bendable crystals should have a slip plane as a prerequisite..$^{27-29}$ Hence, it is hard to modify other properties for applications based on these structures. Surprisingly, the integration of elasticity and luminescent material was realized by researchers. Hayashi and Koizumi successfully combined elasticity with fluorescent property in 1,4-bis[2(4-methylthienyl)]-2,3,5,6-

State Key Laboratory of Supramolecular Structure and Materials, College of Chemistry, Jilin University, Qianjin Street, Changchun, P. R. China. E-mail: hongyuzhang@jlu.edu.cn

$\dagger$ Electronic supplementary information (ESI) available. See DOI: $10.1039 / \mathrm{c} 8 \mathrm{sc} 03135 \mathrm{e}$ tetrafluorobenzene crystals. ${ }^{19,20}$ Compared with traditional luminescent materials that are known to be brittle, the use of elastic organic luminescent crystals could be more flexible. We developed a new kind of elastic self-doping crystals and highlighted the optical applications including optical waveguide and amplified spontaneous emission both in straight-shaped crystals and in bent-shaped crystals. ${ }^{21}$ In addition, recently, the low-loss flexible optical waveguide was also realized by us based on an elastic Schiff base crystal. ${ }^{22}$ These studies significantly expand the value of luminescent materials.

Room-temperature phosphorescence (RTP) has aroused great interests due to its unique photophysical process and promising application value. ${ }^{30-34}$ However, there is no report about flexible crystals with room-temperature phosphorescence. Besides for the limitation of species of flexible organic crystals, we think that there are two additional factors for this situation. The design of RTP materials is the first problem because of the forbidden singlet-to-triplet transition. This problem could be solved by introducing carbonyl $(\mathrm{C}=\mathrm{O})$ groups, hetero atoms or heavy atoms. ${ }^{35-37}$ Other methods including $\mathrm{H}$-aggregation, ${ }^{38}$ crystallization, ${ }^{39,40}$ intermolecular electronic coupling ${ }^{41}$ and so on $^{42,43}$ were also proposed for the construction of RTP materials. In addition to the problem of designing RTP materials, the neglect of crystallographic nature in application also pushes away the value of RTP single crystals. The major reason is that the crystallographic nature of a large single crystal is not suitable for the mainstream applications of RTP materials. To date, the potential applications of RTP materials mainly lie in bio-imaging, lighting, sensors and so on. 
When considering the application of single crystals, amplified spontaneous emission and optical waveguide are usually praised. The phosphorescence nanowire laser was realized by $\mathrm{Fu}$ et al., ${ }^{44}$ and the nanowire phosphorescence optical waveguide was realized by Zhao et al. ${ }^{45}$ To our best knowledge, there is no report on large single RTP crystals displaying similar optical applications.

In this study, we report the single crystals $4,4^{\prime}$-dibromobenzil (DBBZL) with room-temperature phosphorescence as well as controllable elastic and plastic bending. The crystallizationinduced phosphorescence (CIP) of DBBZL has already been reported by Tang et al. ${ }^{46}$ But further photophysical properties and potential applications have not been reported yet. The crystals with suitable size display good elasticity under applied stress. It should be noted that macroscopic elastic bending could turn into macroscopic plastic bending after excessive bending. Detailed discussion revealed that the crystal structure and interactions play a key role in the bending process. Considering the phosphorescent emission and flexible characteristic, optical waveguide is demonstrated in straight-shaped crystals, elastic bent-shaped crystals and plastic bent-shaped crystals. To the best of our knowledge, this study is the first report on the large single RTP crystal that displays optical waveguiding feature.

\section{Results and discussion}

DBBZL is an analytical agent bought from TCI. To further purify the agent, silica gel column chromatography and vacuum thermal sublimation were performed. After slow evaporation of its saturated $\mathrm{CH}_{2} \mathrm{Cl}_{2}$ solution at room temperature, DBBZL crystals with large size and intense phosphorescence are formed. As shown in Fig. 1d, DBBZL displays a green emission spectrum with band maximum at $514 \mathrm{~nm}$. The average lifetime of $279 \mu$ s proves that the emission behavior of DBBZL could be assigned to room-temperature phosphorescence (Fig. 1e). The lifetime is much longer than that of fluorescence which is generally around a few nanoseconds, and thus can be applied in
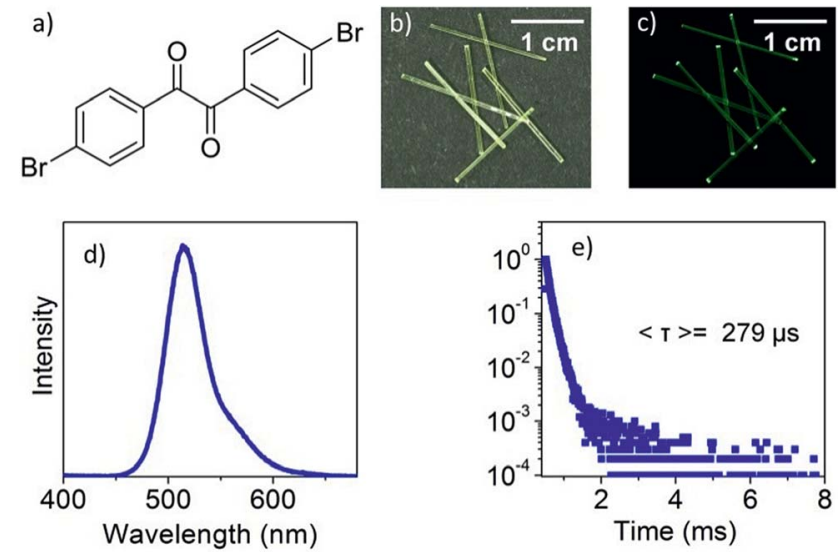

Fig. 1 Molecular structure of DBBZL (a); photographs of single DBBZL crystals taken under daylight (b) and $365 \mathrm{~nm}$ UV light (c); emission spectrum of the DBBZL crystal (d); phosphorescent decay curve of DBBZL measured at $514 \mathrm{~nm}(\mathrm{e})$. the time-gated detection to distinguish from the fluorescence, although the value is short in comparison to the sophisticatedly developed RTP materials. ${ }^{\mathbf{3 0 , 3 4}}$ The crystal structure of DBBZL was then determined by single-crystal X-ray diffraction. The molecule in DBBZL possesses a twist conformation with a dihedral angle of $55.98^{\circ}$ between two benzene rings and connects other molecules through $\mathrm{C}-\mathrm{H} \cdots \mathrm{O}$ and $\mathrm{C}-\mathrm{H} \cdots \pi$ intermolecular interactions (Fig. S2 and S3†). The abundant intermolecular interactions could effectively restrict the intramolecular motions, which help to minimize the nonradiative loss of triplet excitons and promote the phosphorescent emission. ${ }^{39}$

The DBBZL crystal displays good elasticity under applied stress. As shown in Fig. 2a-d, when the crystal was compressed by tweezers, the crystal could easily bend into a semicircle and it also has the ability to bend further. The crystal quickly regained its original straight shape without breaking or cracking after the withdrawal of tweezers (Fig. 2e). This reversible bendingrelaxation process could be repeated many times (Fig. 2f-i), therefore highlighting its good elasticity. The elastic bending process can be intuitively observed in the Movie S1 of ESI. $\dagger$ In the bending process of the DBBZL crystal, the bending plane is associated with the (010) plane (Fig. S4 $\dagger)$. We also tested $(-100)$ plane and applied force, but the crystal directly cracked without bending (Fig. S5 $\dagger$ ). In the layer of (010) plane, as shown in Fig. S6 and $\mathrm{S} 7, \uparrow$ the benzene rings of one molecule are parallel to the benzene rings of other molecules with a distance of 3.47 $\AA$, and the distance between the centroids of benzene rings is 3.93 A. These data appropriately fit to $\pi-\pi$ interaction distance. ${ }^{47-49}$ The molecules form a chain through $\pi-\pi$ stacking and $\mathrm{C}-\mathrm{H} \cdots \mathrm{O}$ hydrogen bonds $(2.7 \AA)$. The chains are connected with each other through $\mathrm{C}-\mathrm{H} \cdots \pi$ and $\mathrm{C}-\mathrm{H} \cdots \mathrm{O}$ interactions, forming a layered structure, which is associated with the crystallographic (010) plane (Fig. 3c and S8†). In the bending process, structural deformations occurred in these layers. In brief, the layer deformation mainly has two types, which are called expansion and contraction (Fig. S9†). This situation demands that the layer structure should have a buffered platform to dissipate energy, thereby promising the $\pi-\pi$ stacking.
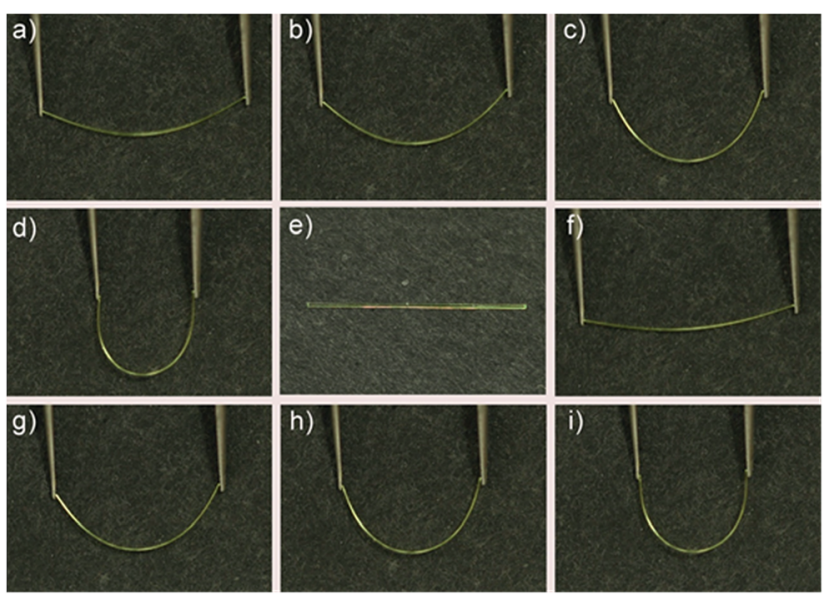

Fig. 2 Photographs of elastic bending process of the DBBZL crystal compressed by tweezers. 
a)

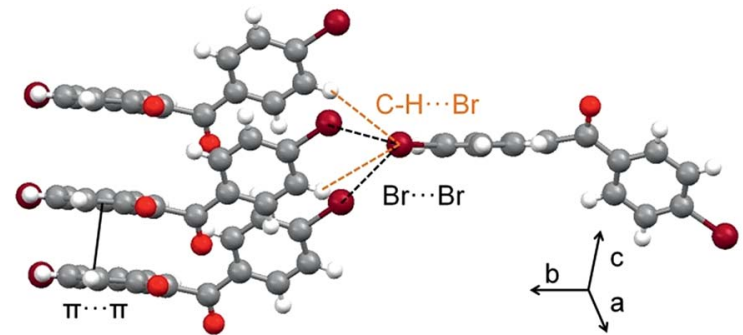

c)

b)

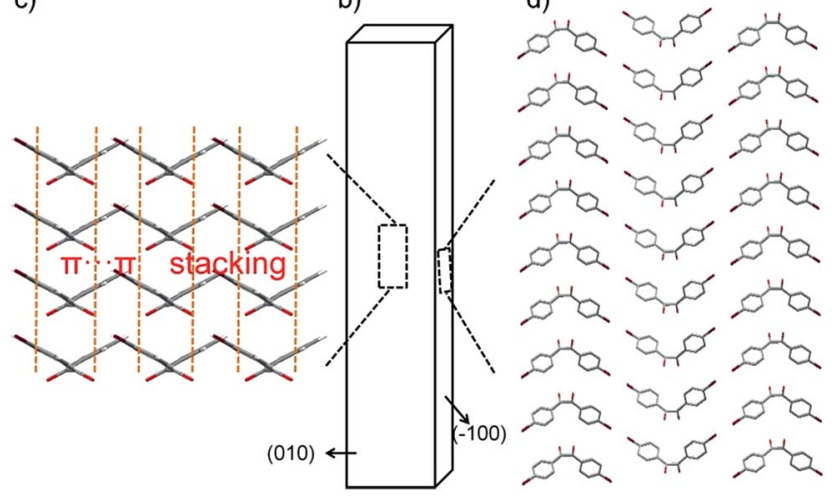

Fig. 3 Crystal packing in DBBZL. Representative supramolecular synthons of the molecules between bending layers (a); crystal morphology with face indices (b), crystal structure viewed down the major bending face (010), the orange lines indicate the $\pi-\pi$ stacking mode (c); and crystal structure viewed down the (-100) face (d).

In the expanding process and contracting process, the $\pi-\pi$ stacking could provide a structural buffering unit for the deformations and it could also be easily recovered. Hence, we conclude that $\pi-\pi$ stacking plays a core role in the elastic bending process.

After excessive bending, elastic crystals are generally broken into two pieces. ${ }^{21,22,26}$ Interestingly, the DBBZL crystals could easily exhibit macroscopic plastic bending if bent excessively. The Movie S2 of ESI $\uparrow$ clearly shows this plastic bending process. Moreover, the plastically bent crystal could be straightened out upon applying stress (Movie S3 of ESI $\dagger$ ). To quantify this deformation process, the bending strain at the end of elastic deformation, which is also the beginning of plastic deformation, was calculated by a simple method. ${ }^{21,22}$ According to the formula of bending strain (see ESI $\dagger$ ), the crystal with decreased thickness could be elastically bent to a loop shape with smaller radius before transforming to plastic bending. The radius of curvature of the bent crystal was controlled by a ballpoint pen refill with a radius of $2.25 \mathrm{~mm}$. Thus, the bending strain value of the thinnest crystal, which exhibits plastic bending, should correspond to the extreme value of elastic bending strain. Finally, a crystal with thickness of $0.122 \mathrm{~mm}$ was calculated with a bending strain value of $2.65 \%$. In other words, the crystals would exhibit macroscopic plastic bending, when the bending strain is over $2.65 \%$.

After excessive bending, the crystals exhibit macroscopic plastic bending (Fig. 4d). Actually, this overbending process (plastic bending) could be divided into three parts: (1) during
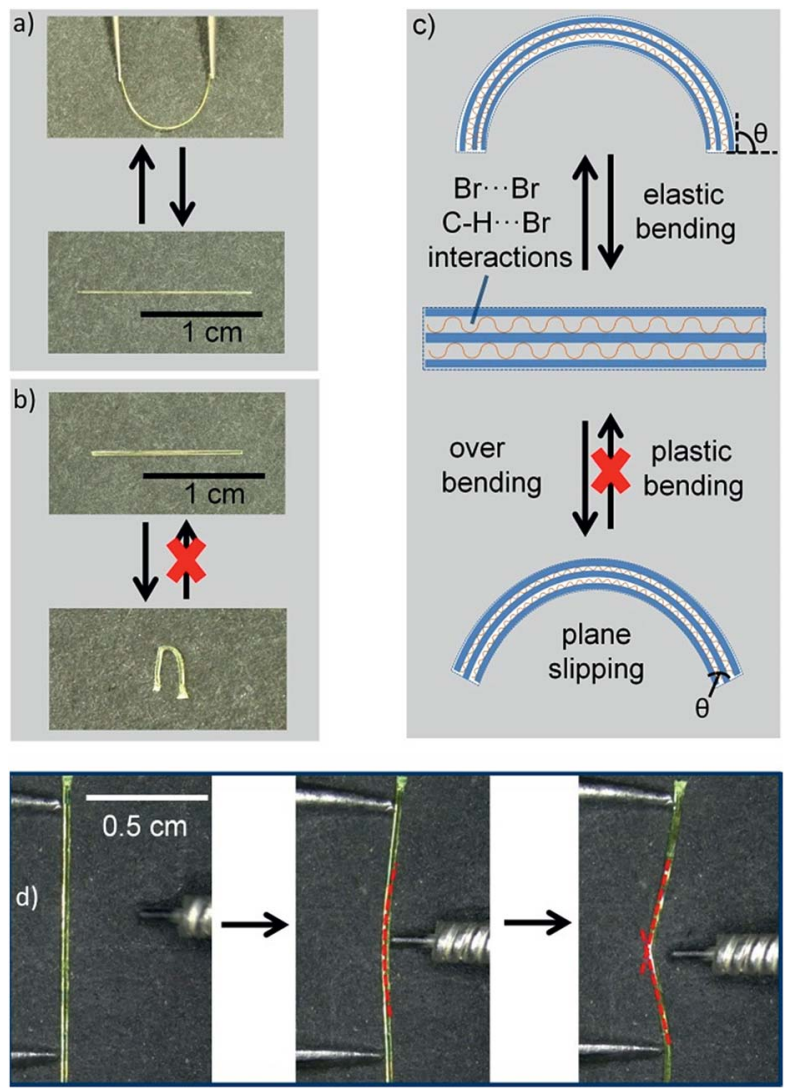

Fig. 4 Photographs of elastic bending (a) and plastic bending (b) under mechanical stress applied through a pair of tweezers; schematic of the crystal before and after elastic or plastic bending (c); and photographs of the overbending process (d).

elastic process, the crystal could form a circular arc shape; (2) the plane slipped when excessive bending occurred; and (3) recovering the bending plane during the slipping process. This means that the bending plane maintains the initial state, neither expansion nor contraction. Therefore, the main factor for plastic bending is the slipping of planes (Fig. 4c). To prove the plastic bending, the interfacial angles of the crystal ends $(\theta)$ attract our attention. The bending faces expand or contract during the elastic bending process and $\theta$ remains unchanged. $^{26-28}$ Compared with elastic bending, the plane slipping in plastic bending makes the bending plane maintain the initial state, but $\theta$ would be changed. The angles between the crystal top and bending faces $(\theta)$ are obviously changed as shown in Fig. S10.† Regarding the effect of crystal thickness on plastic bending, we found that the relatively thinner crystal shows better plastic bending ability and could be folded by about $180^{\circ}$, while the relatively thicker crystal was easily broken due to splitting (Fig. S11 of ESI $\dagger$ ). This splitting further proved that the molecular layer structures were slipped from each other in the plastic bending process.

The elastic bending process demands an isotropic packing mode, whereas the plastic bending process demands an anisotropic packing in which strong and weak interactions exist in nearly perpendicular directions. ${ }^{\mathbf{2 6 - 2 8}}$ Hence, elasticity and 

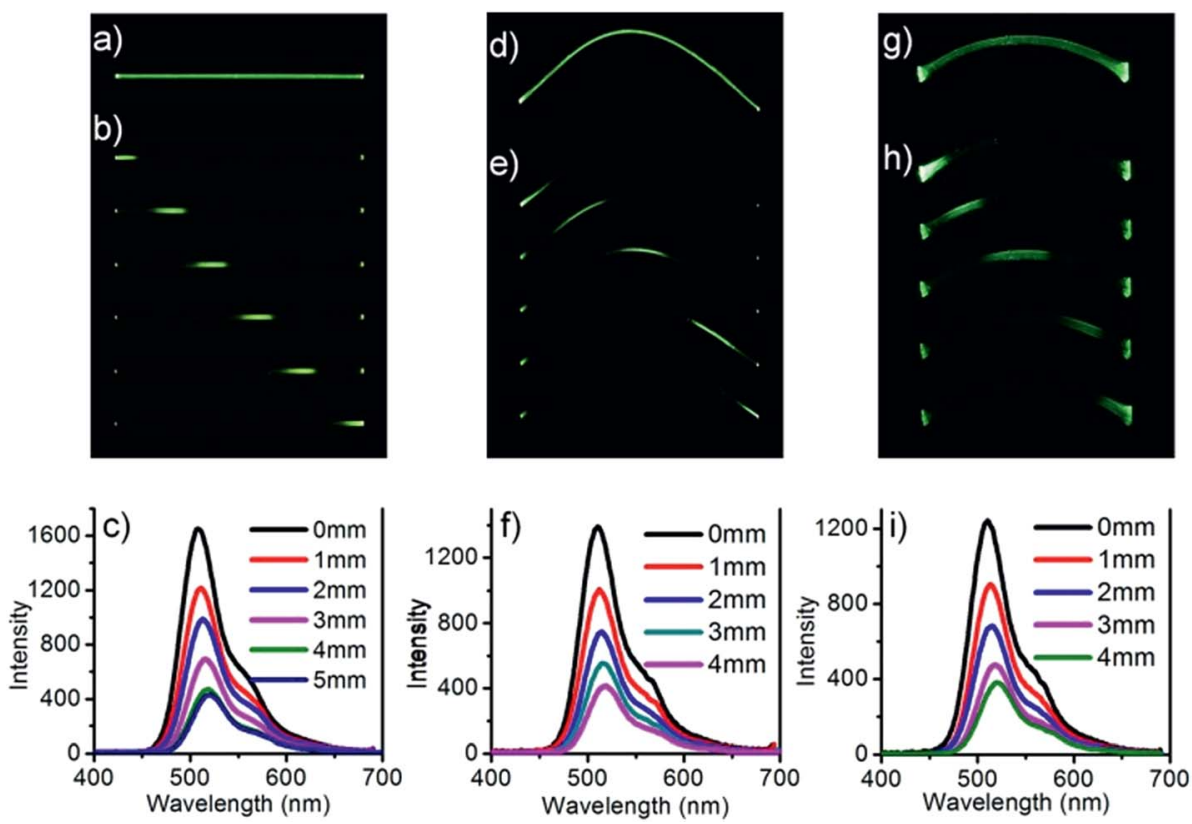

Fig. 5 Phosphorescent optical waveguide properties of single DBBZL crystals in straight shape (a-c), in elastic bent shape (d-f) and in plastic bent shape ( $\mathrm{g}-\mathrm{i})$. Photographs of the crystal under UV light ( $\mathrm{a}, \mathrm{d}$ and g); images collected at different positions of the crystal upon $355 \mathrm{~nm}$ laser ( $\mathrm{b}$, $\mathrm{e}$ and $\mathrm{h}$ ). Phosphorescent spectra measured at one end of the crystal with constantly changing distances between the end and the laser excitation positions ( $c$, $f$ and i).

plasticity are incompatible with each other. But why does DBBZL crystals exhibit controllable elastic and plastic bending? This phenomenon demands the crystal structure to be kept in an intermediate region between anisotropic packing and isotropic packing. This special structure demands that the weak interactions should not be very weak so that they could satisfy at least two conditions: (1) interactions could prevent bending plane from slipping in elastic bending; and (2) interactions could provide chance for slipping in plastic bending. In the crystal structure of $(-100)$ plane, the Br-atoms form type-II $\mathrm{Br} \cdots$ $\mathrm{Br}\left(3.77 \AA, \theta_{1}=94.3^{\circ}, \theta_{2}=162.0^{\circ}\right)$ contacts and $\mathrm{C}-\mathrm{H} \cdots \mathrm{Br}$ interactions (3.14 $\AA$; $3.30 \AA$ ). The bending planes stack in along crystallographic $b$ axis through these interactions (Fig. 3d). The multiple $\mathrm{Br} \cdots \mathrm{Br}$ and $\mathrm{C}-\mathrm{H} \cdots \mathrm{Br}$ interactions (Fig. 3a), which are arranged in different directions and balanced with each other, are relatively stable and thus could avoid changing the position of the molecule, i.e. prevent slipping of the molecular layer structure, in the elastic bending process. Subsequently, the balance of multiple interactions should have several metastable states, i.e. the position of the molecule should be able to change to a certain extent. Thus, the molecular layers can be gently slipped in the plastic bending process. Briefly, the multiple $\mathrm{Br} \cdots$ $\mathrm{Br}$ and $\mathrm{C}-\mathrm{H} \cdots \mathrm{Br}$ interactions endow the crystal with the characters of both isotropic packing (elastic bending) and anisotropic packing (plastic bending).

We further conducted X-ray diffractions of DBBZL crystals in straight shape, elastically bent shape as well as plastically bent shape (Fig. S12 of ESI†ं). In comparison to the straight crystal that shows regularly arranged diffraction peaks, the elastically bent crystal and the plastically bent crystal display elongated and sparse diffraction peaks. Thus, the crystal structures are changed differently via different methods of mechanical bending.

When the DBBZL crystal was exposed to $365 \mathrm{~nm}$ UV irradiation, the emission intensities at both ends were much higher than that in the middle of the crystal. Considering that such crystal often possesses good luminescent intensity and high flexibility, we attempt to combine flexibility with its photophysical properties to enrich the potential applications of RTP materials. Hence, the potential optical application as phosphorescent optical waveguide was first realized both in straightshaped large crystals and in bent-shaped large crystals. We first investigated the optical waveguide in straight shape (Fig. 5a-c). The emission bands were recorded at one end of the crystal upon irradiation by a $355 \mathrm{~nm}$ pulse laser in different positions. The emission intensity at the end of the crystal has a significant inverse relationship with the distance between the irradiated position and the emitting tip. The optical loss coefficient $(\alpha$ in $\left.\mathrm{dB} \mathrm{mm}{ }^{-1}\right)$ was calculated by the formula $I_{\text {tip }} / I_{\text {body }}=A \exp (-\alpha d)$, where $I_{\text {tip }}$ and $I_{\text {body }}$ are the emission intensities at the emitting tip and the irradiated position, respectively, while $d$ is the guiding distance. The calculated optical loss coefficient is around $0.285 \mathrm{~dB} \mathrm{~mm}^{-1}$ (Fig. S13†).

After measuring the straight-shaped crystal, the crystal was bent and fixed with adhesive to investigate the elastic crystal. Surprisingly, the flexible optical waveguide was also realized in the bent crystal, which is shown in Fig. $5 \mathrm{~d}-\mathrm{f}$. The calculated optical loss coefficient is around $0.306 \mathrm{~dB} \mathrm{~mm}^{-1}$. Finally, the plastic crystal was also realized under the same condition, which is shown in Fig. $5 \mathrm{~g}-\mathrm{i}$. The calculated optical loss coefficient is around $0.307 \mathrm{~dB} \mathrm{~mm}^{-1}$. Though the loss coefficients of bent crystals are slightly greater than that of straight-shaped 
crystals, they are still significantly smaller than those of many organic crystals ever reported.50,51 This proved to be a good flexible optical waveguide performance of the single RTP crystals.

\section{Conclusions}

In summary, we report a crystallization-induced phosphorescence DBBZL crystal with controllable elastic and plastic bending. Analysis of the crystal structures suggests that the intermediate region between anisotropic packing and isotropic packing plays a core role in the bending process. In other words, the $\pi-\pi$ stacking is dominant to the elastic bending process, while the $\mathrm{Br} \cdots \mathrm{Br}$ interactions and $\mathrm{C}-\mathrm{H} \cdots \mathrm{Br}$ interactions are dominant to the plastic bending process. Taking advantages of the high flexibility and phosphorescent emission, the first report about flexible optical waveguides of large phosphorescent crystals is realized in straight-shaped crystals, elastic bentshaped crystals and plastic bent-shaped crystals highlighting "elasticity", "plasticity" and "room-temperature phosphorescence". Hence, we believe this work would give a new way to both flexible organic crystals and optical devices.

\section{Conflicts of interest}

There are no conflicts to declare.

\section{Acknowledgements}

This study was supported by the National Natural Science Foundation of China (51622304 and 51773077).

\section{Notes and references}

1 D. P. Karothu, J. Weston, I. T. Desta and P. Naumov, J. Am. Chem. Soc., 2016, 138, 13298.

2 B. Tang, Z. Zhang, H. Liu and H. Zhang, Chin. Chem. Lett., 2017, 28, 2129.

3 P. Naumov, S. Chizhik, M. K. Panda, N. K. Nath and E. Boldyreva, Chem. Rev., 2015, 115, 12440.

4 F. Ding, Y. Zhan, X. Lu and Y. Sun, Chem. Sci., 2018, 9, 4370. 5 A. Nicol, W. Qin, R. T. K. Kwok, J. M. Burkhartsmeyer, Z. Zhu, H. Su, W. Luo, J. W. Y. Lam, J. Qian, K. S. Wong and B. Z. Tang, Chem. Sci., 2017, 8, 4634.

6 E. M. Horstman, R. K. Keswani, B. A. Frey, P. M. Rzeczycki, V. LaLone, J. A. Bertke, P. J. A. Kenis and G. R. Rosania, Angew. Chem., Int. Ed., 2017, 56, 1815.

7 S. Ghosh, M. K. Mishra, S. Ganguly and G. R. Desiraju, J. Am. Chem. Soc., 2015, 137, 9912.

8 S. Saha and G. R. Desiraju, Chem. Commun., 2016, 52, 7676. 9 S. Saha and G. R. Desiraju, Chem. Commun., 2018, 54, 6348.

10 A. K. Saini, K. Natarajan and S. M. Mobin, Chem. Commun., 2017, 53, 9870.

11 M. K. Mishra, K. Mishra, S. A. Syed Asif and P. Manimunda, Chem. Commun., 2017, 53, 13035.

12 N. K. Nath, L. Pejov, S. M. Nichols, C. Hu, N. Saleh, B. Kahr and P. Naumov, J. Am. Chem. Soc., 2014, 136, 2757.
13 S. Ohshima, M. Morimoto and M. Irie, Chem. Sci., 2015, 6, 5746.

14 S. P. Thomas, M. W. Shi, G. A. Koutsantonis, D. Jayatilaka, A. J. Edwards and M. A. Spackman, Angew. Chem., Int. Ed., 2017, 56, 8468.

15 S. Saha and G. R. Desiraju, J. Am. Chem. Soc., 2017, 139, 1975. 16 L. Zhu, R. O. Al-Kaysi and C. J. Bardeen, J. Am. Chem. Soc., 2011, 133, 12569.

17 D. Kitagawa, H. Tsujioka, F. Tong, X. Dong, C. J. Bardeen and S. Kobatake, J. Am. Chem. Soc., 2018, 140, 4208.

18 P. Commins, I. T. Desta, D. P. Karothu, M. K. Panda and P. Naumov, Chem. Commun., 2016, 52, 13941.

19 S. Hayashi and T. Koizumi, Angew. Chem., Int. Ed., 2016, 55, 2701.

20 S. Hayashi, A. Asano, N. Kamiya, Y. Yokomori, T. Maeda and T. Koizumi, Sci. Rep., 2017, 7, 9453.

21 R. Huang, C. Wang, Y. Wang and H. Zhang, Adv. Mater., 2018, 30, 1800814.

22 H. Liu, Z. Lu, Z. Zhang, Y. Wang and H. Zhang, Angew. Chem., Int. Ed., 2018, 57, 8448.

23 Q. H. Cui, Y. S. Zhao and J. Yao, J. Mater. Chem., 2012, 22, 4136.

24 S. Horiuchi and Y. Tokura, Nat. Mater., 2008, 7, 357.

25 Q. H. Cui, Y. S. Zhao and J. Yao, Chem. Sci., 2014, 5, 52.

26 S. Ghosh and C. M. Reddy, Angew. Chem., Int. Ed., 2012, 51, 10319.

27 C. M. Reddy, R. C. Gundakaram, S. Basavoju, M. T. Kirchner, K. A. Padmanabhan and G. R. Desiraju, Chem. Commun., 2005, 3945.

28 C. M. Reddy, K. A. Padmanabhan and G. R. Desiraju, Cryst. Growth Des., 2006, 6, 2720.

29 G. R. Krishna, R. Devarapalli, G. Lal and C. M. Reddy, J. Am. Chem. Soc., 2016, 138, 13561.

30 Y. Xie, Y. Ge, Q. Peng, C. Li, Q. Li and Z. Li, Adv. Mater., 2017, 29, 1606829.

31 B. Xu, H. Wu, J. Chen, Z. Yang, Z. Yang, Y. C. Wu, Y. Zhang, C. Jin, P. Y. Lu, Z. Chi, S. Liu, J. Xu and M. Aldred, Chem. Sci., 2017, 8, 1909.

32 E. Lucenti, A. Forni, C. Botta, L. Carlucci, C. Giannini, D. Marinotto, A. Pavanello, A. Previtali, S. Righetto and E. Cariati, Angew. Chem., Int. Ed., 2017, 56, 16302.

33 Z. Chai, C. Wang, J. Wang, F. Liu, Y. Xie, Y. Z. Zhang, J. R. Li, Q. Li and Z. Li, Chem. Sci., 2017, 8, 8336.

34 W. Zhao, Z. He, J. W. Y. Lam, Q. Peng, H. Ma, Z. Shuai, G. Bai, J. Hao and B. Z. Tang, Chem, 2016, 1, 592.

35 O. Bolton, K. Lee, H. J. Kim, K. Y. Lin and J. Kim, Nat. Chem., 2011, 3, 205.

36 S. Hirata, K. Totani, J. Zhang, T. Yamashita, H. Kaji, S. R. Marder, T. Watanabe and C. Adachi, Adv. Funct. Mater., 2013, 23, 3386.

37 S. Hirata, K. Totani, H. Kaji, M. Vacha, T. Watanabe and C. Adachi, Adv. Opt. Mater., 2013, 1, 438.

38 Z. An, C. Zheng, Y. Tao, R. Chen, H. Shi, T. Chen, Z. Wang, H. Li, R. Deng, X. Liu and W. Huang, Nat. Mater., 2015, 14, 685. 
39 W. Yuan, X. Shen, H. Zhao, J. W. Y. Lam, L. Tang, P. Lu, C. Wang, Y. Liu, Z. Wang, Q. Zheng, J. Sun, Y. Ma and B. Z. Tang, J. Phys. Chem. C, 2010, 114, 6090.

40 Y. Gong, L. Zhao, Q. Peng, D. Fan, W. Yuan, Y. Zhang and B. Z. Tang, Chem. Sci., 2015, 6, 4438.

41 Z. Yang, Z. Mao, X. Zhang, D. Ou, Y. Mu, Y. Zhang, C. Zhao, S. Liu, Z. Chi, J. Xu, Y. C. Wu, P. Y. Lu, A. Lien and M. R. Bryce, Angew. Chem., Int. Ed., 2016, 55, 2181.

42 Z. Cheng, H. Shi, H. Ma, L. Bian, Q. Wu, L. Gu, S. Cai, X. Wang, W. W. Xiong, Z. An and W. Huang, Angew. Chem., Int. Ed., 2018, 57, 678.

43 C. Sun, X. Ran, X. Wang, Z. Cheng, Q. Wu, S. Cai, L. Gu, N. Gan, H. Shi, Z. An, H. Shi and W. Huang, J. Phys. Chem. Lett., 2018, 9, 335.

44 Z. Yu, Y. Wu, L. Xiao, J. Chen, Q. Liao, J. Yao and H. Fu, J. Am. Chem. Soc., 2017, 139, 6376.
45 M. Sun, Y. Liu, Y. Yan, R. Li, Q. Shi, Y. Zhao, Y. Zhong and J. Yao, J. Am. Chem. Soc., 2018, 140, 4269.

46 Y. Gong, Y. Tan, H. Li, Y. Zhang, W. Yuan, Y. Zhang, J. Sun and B. Z. Tang, Sci. China: Chem., 2013, 56, 1183.

47 S. Burattini, B. W. Greenland, D. H. Merino, W. Weng, J. Seppala, H. M. Colquhoun, W. Hayes, M. E. Mackay, I. W. Hamley and S. J. Rowan, J. Am. Chem. Soc., 2010, 132, 12051.

48 S. Sivakova, D. A. Bohnsack, M. E. Mackay, P. Suwanmala and S. J. Rowan, J. Am. Chem. Soc., 2005, 127, 18202.

49 H. M. Colquhoun, D. J. Williams and Z. Zhu, J. Am. Chem. Soc., 2002, 124, 13346.

50 E. Wang, J. W. Y. Lam, R. Hu, C. Zhang, Y. Zhao and B. Z. Tang, J. Mater. Chem. C, 2014, 2, 1801.

51 Y. Li, Z. Ma, A. Li, W. Xu, Y. Wang, H. Jiang, K. Wang, Y. Zhao and X. Jia, ACS Appl. Mater. Interfaces, 2017, 9, 8910. 